\title{
Developing Digital Botanical Resources for Pakistan
}

\author{
Mary Barkworth ${ }^{\ddagger}$, Mushtaq Ahmad ${ }^{\S}$, Mudassir Asrarl, Raza Bhatti", Neil Cobb\#, Lubna Fareed ${ }^{\S}$, \\ Shahid Jamil", Shakeel Khaskheli", Rabia Memon", Abdur Rashid", Amir Sultan`, Zahid Ullah \\ ‡ Utah State University, Logan, United States of America \\ $\S$ Quaid-i-Azam University, Islamabad, Pakistan \\ | University of Balochistan, Quetta, Pakistan \\ II Shah Abdul Latif University, Khairpur, Pakistan \\ \# Northern Arizona University, Flagstaff, United States of America \\ a Post Graduate College Bannu, Bannu, Pakistan \\ « University Sindh Jamshoro, Jamshoro, Pakistan \\ » University of Peshawar, Peshawar, Pakistan \\ ^ National Herbarium of Pakistan, Rawalpindi, Pakistan \\ `University of Swat, Swat, Pakistan
}

Corresponding author: Mary Barkworth (mary.barkworth@usu.edu)

Received: 01 May 2019 | Published: 13 Jun 2019

Citation: Barkworth M, Ahmad M, Asrar M, Bhatti R, Cobb N, Fareed L, Jamil S, Khaskheli S, Memon R, Rashid A,

Sultan A, Ullah Z (2019) Developing Digital Botanical Resources for Pakistan. Biodiversity Information Science and Standards 3: e35885. https://doi.org/10.3897/biss.3.35885

\section{Abstract}

In 2017, funding from the Biodiversity Information Fund for Asia accelerated data mobilization and georeferencing by Pakistani herbaria. The funding directly benefited only two herbaria but, by the end of the project 9 herbaria were involved in sharing data, 2 through GBIF (ISL 2019, SINDH 2019; codes according to Index herbariorum) and 6 others (BANNU 2019, BGH 2019, PUP 2019, QUETTA 2019, RAW 2019, SWAT 2019) through OpenHerbarium, a Symbiota based network. Eventually, all collections in OpenHerbarium are expected to become GBIF data providers. Additional Pakistani herbaria are being introduced to data mobilization and several individuals have expressed interest in learning to use OpenHerbarium to generated documented checklists for teaching and research and others for learning to link information in OpenHerbarium to other resources. These are the first steps to developing a "a large group of individuals ... to train, mentor, and champion [biodiversity] data use" in Pakistan, but it is important to remember that good bioidiversity data starts in the field. We need to provide today's collectors and educators with easy access to a) information about what constitutes a high-quality herbarium specimen; b) tools 
for making it easier to record and provide high quality specimen data; c) simple mechanisms for sharing data in ways that provide immediately useful resources; and d) learning to make use of the data becoming available. OpenHerbarium addresses the third and fourth needs and also makes it simple for collections to become GBIF data providers. This year, the focus will be on first two of the three steps identified. Introduction of the new resources will be used to introduce collectors and educators to the ideas underlying provision of biodiversity data that is fit for use and reuse. When Symbiota2 is functional, OpenHerbarium will be moved to that system. This will encourage development of additional tools for using biodiversity data. All these activities are essential to helping spread understanding of the concepts integral to biodiversity informatics.

It is, of course, possible "to train, build, and champion data use" using data for other parts of the world, or provided by institutions from other parts of the world, but embedding good biodiversity data practices into the fabric of a country's biodiversity education and research activities better benefits the country if a substantial portion of the data is generated from within the country. It also helps to spread knowledge of the country's biodiversity among its students. Consequently, our focus in developing Pakistan's capacity in biodiversity informatics is on engaging collections and collectors in sharing biodiversity data, then helping them discover, use, and create methods for developing the insights needed to encourage wise use of the country's biological resources, and encouraging interaction. This will lead to a "community of practice" within Pakistan that can both benefit from and contribute to an international "community of practice".

\section{Keywords}

biodiversity data mobilization, Pakistan, OpenHerbarium, KeyBase, checklists

\section{Presenting author}

Mary E. Barkworth

\section{Presented at}

Biodiversity_Next 2019

\section{Funding program}

Biodiversity Fund for Asia, Higher Education Commission Pakistan (to Memon), Pakistan Science Foundation (to Asrar). 


\section{References}

- $\quad$ BANNU (2019) Postgraduate College Bannu Herbarium. Occurrence dataset. OpenHerbarium.org URL: http://openherbarium.org/portal/collections/misc/collprofiles.php

- $\quad$ BGH (2019) University of Malakand Botanical Garden and Herbarium. Occurrence dataset. penHerbarium.org. URL: http://openherbarium.org/portal/collections/misc/collprofiles.php? collid $=46$

- ISL (2019) Herbarium of Pakistan. Quaid-i-Azam University Herbarium. Occurrence dataset. URL: https://doi.org/10.15468/bp6jy3

- $\quad$ PUP (2019) Univesity of Peshawar Herbarium. Occurrence dataset. OpenHerbarium.org. URL: http://openherbarium.org/portal/collections/misc/collprofiles. php?collid=46

- $\quad$ QUETTA (2019) University of Balochistan Herbarium. Occurrence dataset. OPenHerbarium.org. URL: http://openherbarium.org/portal/collections/misc/ collprofiles. php?collid=47

- $\quad$ RAW (2019) National Herbarium of Pakistan. Occurrence dataset. OpenHerbarium.org. URL: http://openherbarium.org/portal/collections/misc/collprofiles. php?collid=45

- $\quad$ SINDH (2019) Institute of Plant Sciences. University of Sindh Jamshoro Herbarium. Occurrence dataset. GBIF URL: https://doi.org/10.15468/vpgzhd

- $\quad$ SWAT (2019) University of Swat Herbarium. Occurrence dataset. OpenHerbarium.org. URL: http://openherbarium.org/portal/collections/misc/collprofiles. php?collid=33 